\title{
Unrestricted accessibility of short oligonucleotides to RNA
}

\author{
HOWARD B. GAMPER JR., ${ }^{1}$ KHALIL ARAR, ${ }^{2}$ ALAN GEWIRTZ, ${ }^{3}$ and YA-MING HOU ${ }^{1}$ \\ ${ }^{1}$ Department of Biochemistry and Molecular Pharmacology, Thomas Jefferson University, Philadelphia, Pennsylvania, 19107, USA \\ ${ }^{2}$ Proligo LLC, Paris, 75011, France \\ ${ }^{3}$ Division of Hematology/Oncology, University of Pennsylvania School of Medicine, Philadelphia, Pennsylvania 19104, USA
}

\begin{abstract}
The propensity of RNA to fold into higher-order structures poses a major barrier to the use of short probes ( $<15$ nucleotides) by preventing their accessibility. Introduction of the pseudo-complementary bases 2-aminoadenine (nA) and 2-thiouracil (sU) and the destabilizing base 7-deazaguanine (cG) into RNA provides a partial solution to this problem. While complementary in hydrogen bonding groups, $\mathrm{nA}$ and sU cannot form a stable base pair due to steric hindrance, and are thus pseudo-complementary. Each, however, recognizes the regular T/U and A complements, allowing pairing with oligonucleotides. Short pseudocomplementary RNAs can be prepared by in vitro transcription. Relative to standard transcripts, the modified transcripts possess reduced secondary structure and increased accessibility to short (8-mer) probes in the locked nucleic acid (LNA) configuration. They also hybridize to complementary probes with increased specificity and thermostability. Practical application of this strategy to oligonucleotide-based hybridization assays will require engineering of RNA polymerase for more efficient utilization of pseudo-complementary nucleoside triphosphates.
\end{abstract}

Keywords: RNA; LNA; pseudo-complementary; secondary structure; hybridization; 2-aminoadenine; 2-thiouracil; 7-deazaguanine

\section{INTRODUCTION}

Higher-order structures of RNA (and to a lesser extent single-stranded DNA) have severely impeded sequence detection by short probes of 8-10 nucleotides (nt) in length (Doty et al. 1959; Lima et al. 1992; Hacia et al. 1998). This limitation has prevented their general use as specific agents for detection of single nucleotide polymorphisms and point mutations (Wallace et al. 1979; Zoller and Smith 1983), and has posed a serious block to the development of universal arrays for application in the resequencing of genes or the profiling of genetic samples. Even high-affinity probes, such as those with an LNA backbone (Jepsen et al. 2004), lack a general and dependable accessibility to RNA.

We have recently shown (Gamper et al. 2004) that accessibility of short (12-mer) DNA probes to an RNA target can be improved if the RNA is substituted with the pseudocomplementary bases nA (also known as 2,6-diaminopu-

Reprint requests to: Howard B. Gamper Jr., Department of Biochemistry and Molecular Pharmacology, Room 222BLSB, Thomas Jefferson University, 233 South 10th Street, Philadelphia, Pennsylvania, 19107, USA; e-mail: Howard.Gamper@jefferson.edu; fax: (215) 503-4954.

Article and publication are at http://www.rnajournal.org/cgi/doi/ 10.1261/rna.2670705. rine) and $\mathrm{sU}$ and the destabilizing base $\mathrm{hX}$ (hypoxanthine, inosine) (Fig. 1). While these substitutions eliminated intramolecular $\mathrm{A}-\mathrm{U}$ pairing (between $\mathrm{nA}$ and $\mathrm{sU}$ ) and G-U pairing (between $\mathrm{G}$ and $\mathrm{sU}$ ) and attenuated the strength of G-C pairing (between $\mathrm{hX}$ and $\mathrm{C}$ ), they did not completely remove RNA structures to allow unrestricted accessibility to short probes. Furthermore, because basepairing with $\mathrm{hX}$ is degenerate, this modification reduced the specificity of hybridization and was not desirable. In pursuit of a more dependable hybridization technology, we investigated here the possibility that the accessibility problem of RNA could be overcome by substituting the RNA target with the pseudo-complementary bases $\mathrm{nA}$ and $\mathrm{sU}(\mathrm{nA}+\mathrm{sU})$, and the more specific and yet destabilizing base cG (7-deazaguanine). Our results demonstrate that RNA substituted in this way, together with short probes (8-mers) in the LNA configuration, can produce a powerful combination that permits hybridization at essentially all sequences.

\section{RESULTS}

A perfect RNA hairpin (Fig. 2; HP25) of 25 bp, high in G/C content, was tested as a model substrate for the accessibility of probes. This RNA, previously designed and studied by us 


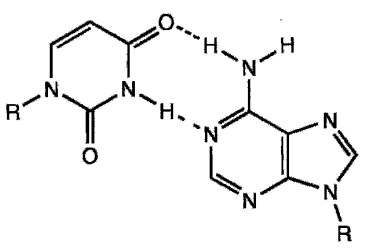

Uracil : Adenine<smiles></smiles>

2-Thiouracil : Adenine

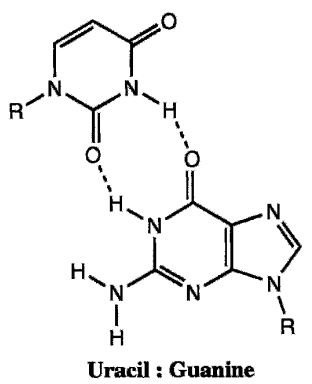

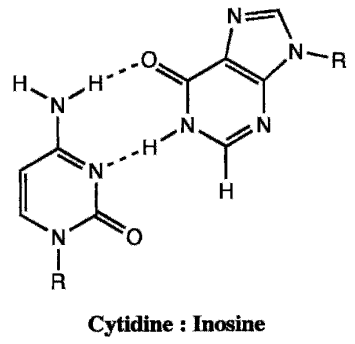

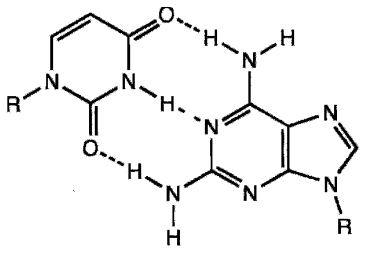

Uracil : 2-Aminoadenine

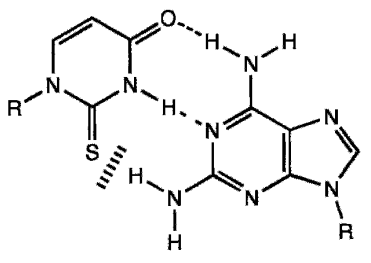

2-Thiouracil : 2-Aminoadenine

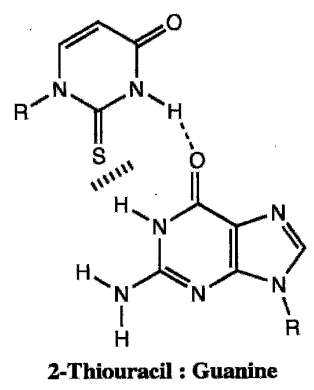

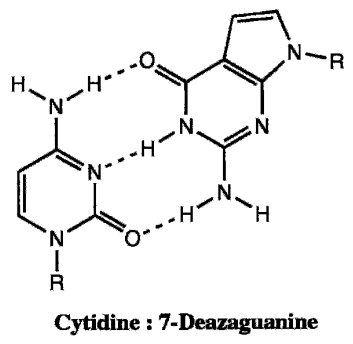

FIGURE 1. Pairing properties of 2-aminoadenine (nA), 2-thiouracil $(\mathrm{sU}), 7$-deazaguanine $(\mathrm{cG})$, and hypoxanthine (hX).

(Gamper et al. 2004), was prepared by in vitro transcription with T7 RNA polymerase, in the presence of the pseudocomplementary $\mathrm{nA}+\mathrm{sU}$ and/or the destabilizing $\mathrm{hX}$ or $\mathrm{cG}$ nucleoside triphosphates (Table 1). These modified nucleoside triphosphates attenuated the transcription of radiolabeled hairpin under standard reaction conditions. Replacement of ATP with nATP and UTP with sUTP reduced the yield of fulllength hairpin by 30\%-40\% with one substitution and by $60 \%-70 \%$ with both substitutions. Replacement of GTP by cGTP had no effect on transcription, but substitution with hXTP reduced the yield of hairpin by almost $75 \%$. Transcription in the presence of all three NTP analogs reduced hairpin synthesis to only $30 \%$ of the control reaction, regardless of whether cGTP or hXTP was used. While these levels of transcription efficiency were adequate for this study, the preparation of pseudo-complementary transcripts longer than $200 \mathrm{nt}$ was problematic (Gamper et al. 2004).

The strength of intramolecular base-pairing was evaluated by the melting temperature $\left(T_{m}\right)$ of each modified hairpin. If the hairpin became destabilized because of the presence of modified bases, the $T_{m}$ of the hairpin should decrease relative to that of the unmodified RNA. The $T_{m}$ was determined by a recently developed gel mobility shift assay (Gamper et al. 2004), where the accessibility of a 25mer RNA probe to the RNA hairpin was monitored by gel electrophoresis under nondenaturing conditions. Determination of the accessibility over a range of temperatures $\left(10^{\circ} \mathrm{C}-90^{\circ} \mathrm{C}\right)$ yielded a binding curve from which the $T_{m}$ was calculated (Table 2). Notably, the hairpin composed of regular bases was not accessible to the probe under any conditions, demonstrating the stability of the hairpin, which was predicted by Mfold to have a $T_{m}$ of $105^{\circ} \mathrm{C}$ in $25 \mathrm{mM} \mathrm{NaCl}$ and $5 \mathrm{mM} \mathrm{MgCl}_{2}$ (Zuker 2003). In contrast, each of the modified hairpins had an experimentally determined $T_{m}$ that was much decreased, indicating destabilization of the hairpin structure. Specifically, substitution with $\mathrm{hX}, \mathrm{cG}$, or $\mathrm{nA}+\mathrm{sU}$ each lowered the $T_{m}$ to $56^{\circ} \mathrm{C}, 77^{\circ} \mathrm{C}$, or $81^{\circ} \mathrm{C}$, respectively, while substitution with $\mathrm{nA}+\mathrm{sU}$ together with $\mathrm{hX}$ or $\mathrm{cG}$ lowered the $T_{m}$ further to $39^{\circ} \mathrm{C}$ or $63^{\circ} \mathrm{C}$. Thus, all of the modified bases reduced the hairpin stability. The denaturing effects of these modified bases were relatively the same in all of the solutions conditions tested, including those lacking $\mathrm{MgCl}_{2}$ or containing formamide. Although the combination of $\mathrm{nA}+\mathrm{sU}$ and $\mathrm{hX}$ appeared to be most effective in $T_{m}$ reduction, $\mathrm{hX}$ is not favorable due to its lack of specificity in base-pairing (see below). Focus is therefore placed on $n A+s U$ and $c G$ to evaluate the ability of this combination to improve the accessibility and specificity of target-probe hybridization.

Probes with an LNA backbone, as short as 7-8 bases, have been shown to hybridize to their target sequences with exquisite stability and specificity (Jepsen et al. 2004). We have confirmed that these properties are retained upon hybridization of LNA probes to a pseudo-complementary RNA. Specifically, the stability of a 7 -mer LNA probe $\left(5^{\prime}-\right.$

$$
\begin{aligned}
& \text { SS14 5'-AAAGCAGACUUCUC } \\
& \text { SS32 5'-GGGAAAAGCAGACUUCUCCUCAGGAGUCAGGU } \\
& \sqrt{\int \mathrm{G}=-5.6 \mathrm{kcal} / \mathrm{mole}} \\
& \text { 5.-GGGA AAG } C_{A} \\
& \text { UGGACUGAGGACن் } \dot{C} \dot{C} \dot{U}_{C} \dot{U}^{\mathrm{U}} \dot{C}_{\mathrm{A}} \mathrm{G}
\end{aligned}
$$

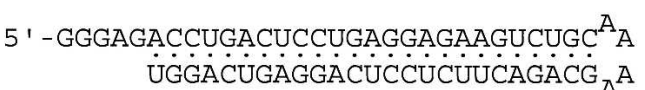

FIGURE 2. RNA targets used in this study. SS14 was chemically synthesized. SS32 and HP25 were transcribed off synthetic DNA templates using different combinations of NTPs. One arm of the HP25 hairpin is recapitulated in SS32. 
TABLE 1. Incorporation of NTP analogs by T7 RNA polymerase

\begin{tabular}{lc}
\hline NTPs & Relative amount of transcript \\
\hline ATP,UTP,GTP,CTP & 1.00 \\
nATP,UTP,GTP,CTP & 0.59 \\
ATP, sUTP,GTP,CTP & 0.70 \\
nATP,sUTP,GTP,CTP & 0.44 \\
ATP,UTP,hXTP,CTP & 0.45 \\
ATP,UTP, CGTP,CTP & 0.98 \\
nATP,sUTP,hXTP,CTP & 0.27 \\
nATP,sUTP,CGTP,CTP & 0.35 \\
\hline
\end{tabular}

A 59-mer RNA transcript (HP25) was generated overnight under standard conditions using a synthetic DNA template that contained the consensus promoter for T7 RNA polymerase. NTPs were present at $1.0 \mathrm{mM}$ except for $\alpha-{ }^{32} \mathrm{P}-\mathrm{CTP}$, which was at $0.5 \mathrm{mM}$. (nA) 2-aminoadenosine, $(\mathrm{sU})$ 2-thiouridine, (hX) hypoxanthine (inosine), (cG) 7-deazaguanosine.

GAAGTCT) hybridized to a pseudo-complementary RNA (Fig. 2; SS32) was assessed by the $T_{m}$ of the hybrid in three different solutions (Table 3). Relative to the hybrid with the unmodified RNA $\left(T_{m}=54^{\circ} \mathrm{C}\right.$ in $20 \%$ formamide), the introduction of $\mathrm{nA}+\mathrm{sU}$ increased the stability of the probe-RNA hybrid by raising the $T_{m}$ to $84^{\circ} \mathrm{C}$. This was expected because $\mathrm{nA}-\mathrm{T} / \mathrm{U}$ and $\mathrm{sU}-\mathrm{A}$ base pairs are known to stabilize the A-form duplex (Vormbrock et al. 1974; Howard and Miles 1984; Kumar and Davis 1997; Testa et al. 1999). Hybrids in which the RNA was substituted with $\mathrm{nA}+\mathrm{sU}$, together with $\mathrm{hX}$ or $\mathrm{cG}$, had $T_{m} \mathrm{~s}$ of $67^{\circ} \mathrm{C}$ and $80^{\circ} \mathrm{C}$, respectively. The reduced $T_{m} \mathrm{~s}$, compared to that with $\mathrm{nA}+\mathrm{sU}$ only, reflect the destabilizing effects of $\mathrm{hX}$ and $\mathrm{cG}$ (Martin et al. 1985; Seela and Driller 1985), as evidenced when each was separately substituted into the RNA strand of the hybrid $\left(T_{m}=29^{\circ} \mathrm{C}\right.$ and $45^{\circ} \mathrm{C}$, respectively). Importantly, despite the destabilizing effect of $\mathrm{cG}$, its combination with $\mathrm{nA}+\mathrm{sU}$ significantly improved the $T_{m}$ of the hybrid $\left(80^{\circ} \mathrm{C}\right)$ relative to the unmodified control $\left(54^{\circ} \mathrm{C}\right)$. Remarkably, these elevated $T_{m}$ values were observed even under stringent conditions $(20 \mathrm{mM}$ HEPES at $\mathrm{pH} 7.5,25 \mathrm{mM}$ $\mathrm{NaCl}, 20 \%$ formamide), reflecting the ability of pseudocomplementary RNA to form extremely stable hybrids with LNA probes.

Specificity of the LNA probes was confirmed by testing their ability to discriminate against mismatches with the pseudo-complementary RNA. One perfect-match and 12 single-mismatch 8-mer LNA probes were each hybridized to single-stranded RNA (SS14 or SS32) that was prepared with different combinations of bases (Fig. 3). The equilibrium between the free RNA and RNA-LNA hybrid was fixed at $8^{\circ} \mathrm{C}$ below the $T_{m}$ of each perfect-match hybrid and then determined by gel shift assay. The fraction of the RNA in the hybrid was analyzed and compared. Here, while the unmodified RNA was permissive for U-G formation, promoting $81 \%$ hybridization (panel 1), substitution of the RNA with $\mathrm{sU}$ prevented this mismatch, promoting only 9\%-12\% hybridization (panels $3-5$ ). This is due to the fact that the sU-G pairing is less stable than U-G (Vormbrock et al. 1974; Testa et al. 1999). Thus, the presence of sU in the RNA increased the ability of the LNA probe to discriminate against the U-G mismatch. Relative to the unmodified target, suppression of the U-T mismatch was much improved for all four modified transcripts, an effect attributable to substitution with $\mathrm{sU}$ or cG. In contrast, presence of $\mathrm{nA}$ in the RNA strand reduced specificity by accommodating an nA-C mismatch, as has been observed in other LNA-containing hybrids (Koshkin 2004; Rosenbohm et al. 2004). Importantly, while substitution with hX decreased the specificity of hybrid formation (panel 5), as expected due to the degeneracy of $\mathrm{hX}$ in base-pairing (Martin et al. 1985; Case-Green and Southern 1994), introduction of $\mathrm{cG}$ retained the specificity (panels 2 and 4), allowing only the cG-C base-pairing. Overall, despite the reduced specificity by $\mathrm{nA}$, the RNA that contained $\mathrm{nA}+\mathrm{s} \mathrm{U}$, alone or in combination with $\mathrm{cG}$, exhibited the greatest selectivity in hybridization.

We also verified that substitution of $\mathrm{nA}+\mathrm{sU}$ and $\mathrm{cG}$ into a structured RNA indeed increases its accessibility to a short LNA probe. An RNA transcript (Fig. 2; SS32) that embodied one arm of the 25-bp-long hairpin described earlier (Gamper et al. 2004) was used as a target. This RNA, in the absence of modification, contained a hairpin at one end that was inaccessible to 12-mer DNA probes at $10^{\circ} \mathrm{C}$ in the presence of $5 \mathrm{mM} \mathrm{MgCl} 2$ and $25 \mathrm{mM} \mathrm{NaCl}$ (Gamper et al. 2004). Here, under identical conditions, an 8-mer LNA probe targeted to the same end was shown to slowly hybridize to the unmodified transcript (Fig. 4). The time course of the hybridization was compared among RNAs substituted with $\mathrm{nA}+\mathrm{sU}$ and/or $\mathrm{hX} / \mathrm{cG}$, to determine the hierarchy of accessibility. Clearly, substitution with $\mathrm{nA}+\mathrm{sU}+\mathrm{cG}$ generated the greatest burst of hybrid, followed by substitution with $\mathrm{nA}+\mathrm{sU}+\mathrm{hX}$. This comparison indicates that stability of the intermolecular LNARNA hybrid can sometimes be more important than destabilization of RNA secondary structure in driving hybrid-

TABLE 2. Melting temperatures of a 25-bp RNA hairpin

\begin{tabular}{lrrrr}
\hline & \multicolumn{5}{c}{ Solution } \\
\cline { 2 - 5 } Base composition of RNA hairpin & 1 & 2 & 3 & 4 \\
\hline $\mathrm{A}, \mathrm{U}, \mathrm{G}, \mathrm{C}^{1}$ & $>100^{\circ}$ & $>100^{\circ}$ & - & - \\
$\mathrm{A}, \mathrm{U}, \mathrm{hX}, \mathrm{C}^{2}$ & $56^{\circ}$ & $46^{\circ}$ & $32^{\circ}$ & $24^{\circ}$ \\
$\mathrm{A}, \mathrm{U}, \mathrm{CG}, \mathrm{C}^{2}$ & $77^{\circ}$ & $71^{\circ}$ & $60^{\circ}$ & $53^{\circ}$ \\
$\mathrm{nA}, \mathrm{SU}, \mathrm{G}, \mathrm{C}^{2}$ & $81^{\circ}$ & $80^{\circ}$ & $70^{\circ}$ & $62^{\circ}$ \\
$\mathrm{nA}, \mathrm{sU}, \mathrm{hX}, \mathrm{C}^{2}$ & $39^{\circ}$ & $41^{\circ}$ & $27^{\circ}$ & $16^{\circ}$ \\
$\mathrm{nA}, \mathrm{SU}, \mathrm{CG}, \mathrm{C}^{2}$ & $63^{\circ}$ & $65^{\circ}$ & $52^{\circ}$ & $45^{\circ}$ \\
\hline
\end{tabular}

The 25-bp RNA hairpin (HP25) was melted in $25 \mathrm{mM} \mathrm{NaCl}, 20 \mathrm{mM}$ HEPES buffer ( $\mathrm{pH} 7.5$ ) with (1) $5 \mathrm{mM} \mathrm{MgCl}_{2}$, (2) no additions, (3) $20 \%$ formamide, or (4) $35 \%$ formamide.

${ }^{1}$ Predicted $T_{m}$ s from Mfold.

${ }^{2} T_{m}$ s determined by gel mobility shift assay using a 25-mer RNA probe (5'-ACCUGACUCCUGAGGAGAAGUCUGC). 
TABLE 3. Melting temperatures of a 7-bp RNA-LNA hybrid

\begin{tabular}{lrrr}
\hline & \multicolumn{3}{c}{ Solution } \\
\cline { 2 - 4 } Base composition of RNA strand & 1 & 2 & 3 \\
\hline $\mathrm{A}, \mathrm{U}, \mathrm{G}, \mathrm{C}$ & $64^{\circ}$ & $58^{\circ}$ & $54^{\circ}$ \\
$\mathrm{A}, \mathrm{U}, \mathrm{hX}, \mathrm{C}$ & $47^{\circ}$ & $37^{\circ}$ & $29^{\circ}$ \\
$\mathrm{A}, \mathrm{U}, \mathrm{CG}, \mathrm{C}$ & $63^{\circ}$ & $55^{\circ}$ & $45^{\circ}$ \\
$\mathrm{nA}, \mathrm{SU}, \mathrm{G}, \mathrm{C}$ & $>90^{\circ}$ & $>90^{\circ}$ & $84^{\circ}$ \\
$\mathrm{nA}, \mathrm{SU}, \mathrm{hX}, \mathrm{C}$ & $80^{\circ}$ & $75^{\circ}$ & $67^{\circ}$ \\
$\mathrm{nA}, \mathrm{SU}, \mathrm{CG}, \mathrm{C}$ & $>90^{\circ}$ & $\sim 90^{\circ}$ & $80^{\circ}$ \\
\hline
\end{tabular}

The RNA-LNA hybrids consisted of a 7-mer LNA probe (5'GAAGTCT) annealed to the unmodified 14-mer RNA target (SS14) or to the 32-mer RNA target (SS32) that was substituted with one or more base analogs. The hybrids were melted in $25 \mathrm{mM}$ $\mathrm{NaCl}, 20 \mathrm{mM}$ HEPES buffer ( $\mathrm{pH}$ 7.5) with (1) $5 \mathrm{mM} \mathrm{MgCl}_{2}$, (2) no additions, or (3) $20 \%$ formamide. $T_{m} s$ were determined by gel mobility shift assay using an 8-mer LNA competitor (5' CAGACTTC).

ization. Further support is provided by the observation that, while substitution of the RNA with $\mathrm{nA}+\mathrm{sU}$ or $\mathrm{cG}$ alone resulted in improved rates of hybridization, substitution with hX alone was insufficient. The LNA 8-mer used here was part of a set of LNAs that was tiled across the RNA target. All members of this set readily hybridized to the unmodified RNA under more stringent conditions (data not shown).

Additional experiments established that pseudo-complementary RNA is fully accessible to short high-affinity probes. Here we used the RNA hairpin presented in Figure 2 (HP25) as the target for a set of tiled 8-mer LNA probes (Fig. 5A). The hairpin was substituted with $\mathrm{nA}+\mathrm{sU}$ and/or $\mathrm{hX} / \mathrm{cG}$, and hybridization was carried out at a temperature $13^{\circ} \mathrm{C}$ above the $T_{m}$ for each hairpin, after which the RNALNA hybrid was kept in an ice bath until examined by gel electrophoresis. These analyses showed that hairpins substituted with $\mathrm{nA}+\mathrm{sU}$ and either $\mathrm{cG}$ or $\mathrm{hX}$ had the ability to hybridize to every member of the LNA probe set, which included a total of 11 oligomers encompassing one arm of the hairpin stem. Hairpin substituted with just $n A+s U$ performed nearly as well, with hybridization observed for nine of the 11 probes. In contrast, hairpin substituted with the highly destabilizing $\mathrm{hX}$ base only hybridized effectively to two probes. Under no conditions did the unmodified hairpin or the hairpin substituted with just $c G$ hybridize to any of the probes (data not shown). The high $T_{m}$ of these hairpins precluded access to LNA probes.

Many of the hybridization reactions analyzed by gel mobility shift assay in Figure 5A generated two or more shifted bands, especially when the hairpin target contained $\mathrm{nA}+\mathrm{sU}$ substitutions. We surmise that multiple bands observed in a single lane may reflect different secondary structures of the same probe-target complex. Since gel electrophoresis was conducted under conditions where each modified hairpin existed in a collapsed state, it is plausible that any one of the hairpins could fold into alternative structures influenced by the position of the LNA-RNA hybrid on the RNA sequence. Formation of mismatched LNA-RNA hybrids might also account for some of the bands, since the hybridization conditions were not that stringent.

Besides substitution of the RNA hairpin with $\mathrm{nA}+$ $\mathrm{s} \mathrm{U}+\mathrm{cG}$, successful and readily accessible hybridization required probes with an LNA backbone. For example, a set of 12-mer DNA probes performed poorly due to their low affinity for the target hairpins (Fig. 5B). Only six of the 14 DNA probes formed a stable hybrid when the hairpin contained $\mathrm{nA}+\mathrm{sU}+\mathrm{hX}$, and none hybridized when it contained $\mathrm{nA}+\mathrm{sU}+\mathrm{cG}$. The DNA-RNA hybrids formed by these probes were stable at $40^{\circ} \mathrm{C}$ but not at $65^{\circ} \mathrm{C}$, thus

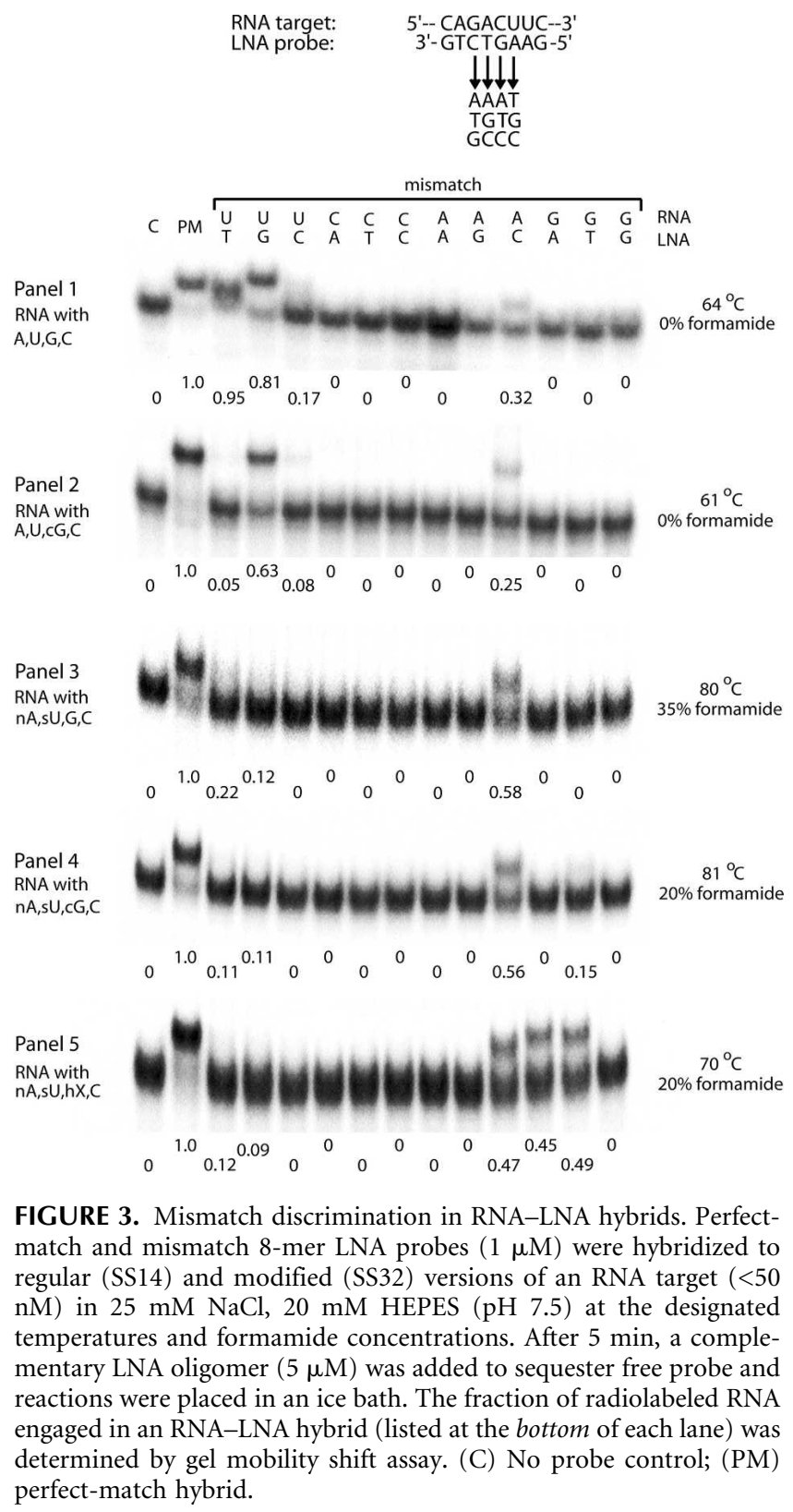




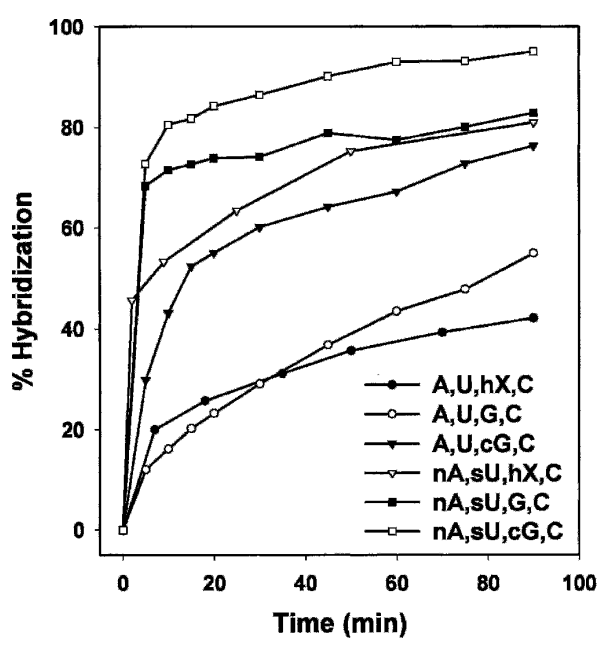

FIGURE 4. Hybridization of an 8-mer LNA probe to a short RNA hairpin element. Radiolabeled SS32 RNA target substituted with the indicated bases was hybridized at $10^{\circ} \mathrm{C}$ to an 8-mer LNA probe (5'-GAAGTCTG; $1 \mu \mathrm{M}$ ) in $5 \mathrm{mM} \mathrm{MgCl}_{2}, 25 \mathrm{mM} \mathrm{NaCl}, 20 \mathrm{mM}$ HEPES ( $\mathrm{pH}$ 7.5). The time course of annealing was determined by adding a competitor LNA oligomer $\left(10 \mu \mathrm{M} ; 5^{\prime}\right.$-CAGACTTC) to replicate reactions at the indicated times. Probe-target hybrid was resolved from target by gel mobility shift assay (Gamper et al. 2004).

precluding hybridization to the cG-containing RNA target. In the gel corresponding to this target, neither full-length hairpin nor prematurely terminated "half-hairpin" were shifted to slower moving bands. The single-stranded halfhairpin target was homologous to the DNA probes and functioned as a control for nonspecific hybridization. Hybrids formed with the hX-containing RNA target, particularly those located near the $3^{\prime}$-end of the RNA, were probably lost upon cooling and subsequent collapse of the RNA hairpin by a strand displacement mechanism. Upstream hybrids escaped this fate by blocking association of the RNA arms that flanked the hairpin loop.

LNA probes formed sufficiently stable hybrids with the pseudo-complementary RNA hairpins to resist strand displacement at temperatures below the melting point of the hairpin. We confirmed this behavior by showing that a 25-mer DNA was unable to displace a 9-mer LNA from a complementary 32-mer RNA (Fig. 6). Gel analysis indicated that a ternary LNA-RNA-DNA complex formed when DNA probe was added after LNA probe to the target RNA. Although the longer DNA probe hybridized to the same sequence as the LNA probe on the RNA target, the single-stranded DNA overhang of the 25-mer probe was unable to displace the LNA. To our knowledge, this is the first example of a short hybrid resistant to branch migration. In this model experiment the weakly hybridizing DNA probe mimicked the inability of the pseudo-complementary RNA hairpin to displace LNA probes. As expected, the 9-mer LNA was displaced from the RNA target by a 25 -mer RNA probe. In the same experiment, short DNA and RNA probes were also displaced from the RNA target by longer overlapping DNA and RNA probes.

\section{DISCUSSION}

Unrestricted accessibility of short probes to RNA requires that the probes be in the LNA configuration and that the RNA target contain the pseudo-complementary $\mathrm{nA}+\mathrm{sU}$ and the destabilizing cG bases. Key to this success was the exceptionally high affinity of the LNA probes to the modified RNA. The RNA-LNA hybrids described here exhibit high thermostability, even under the stringent conditions that denature the pseudo-complementary RNA target. Furthermore, once formed, the stability of these hybrids is such that they are resistant to strand displacement, even at

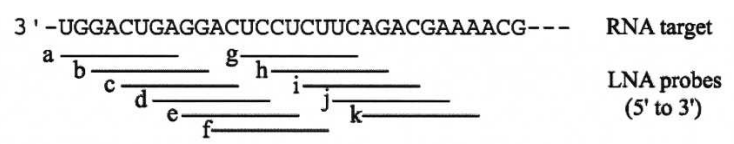

\section{A}
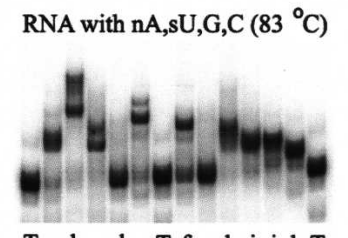

T a b c d e T f g h i j k T

RNA with $\mathrm{nA}, \mathrm{sU}, \mathrm{hX}, \mathrm{C}\left(40^{\circ} \mathrm{C}\right)$

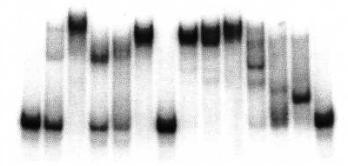

T a b c d e T f g h i j k T

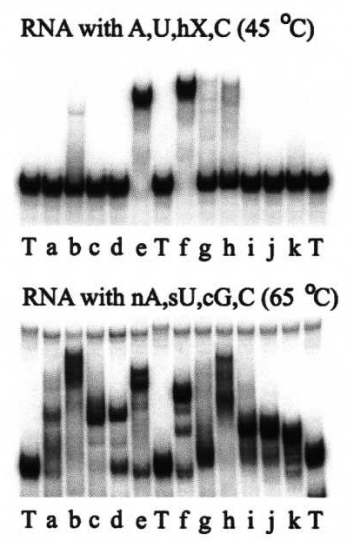

B

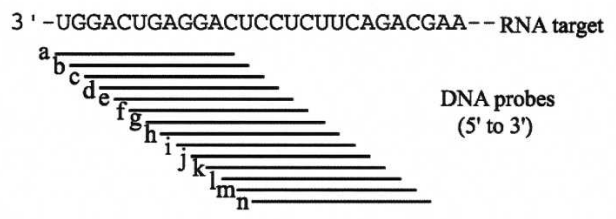

RNA with nA,sU,hX,C ( $\left.40^{\circ} \mathrm{C}\right)$

RNA with nA,sU,cG,C $\left(65^{\circ} \mathrm{C}\right)$

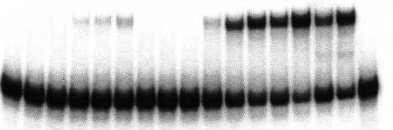

T a b c def g T h i j k l mn T

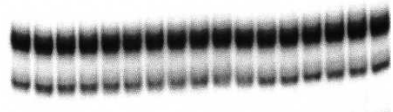

T a b c defg Thi j k l mn T
FIGURE 5. Accessibility of a 25-bp RNA hairpin to short LNA and DNA probes. (A) Hybridization of tiled 8-mer LNA probes to radiolabeled HP25. The probes $(5 \mu \mathrm{M})$ were hybridized at the designated temperatures in $25 \mathrm{mM} \mathrm{NaCl}, 20 \mathrm{mM}$ HEPES ( $\mathrm{pH} \mathrm{7.5),20 \%} \mathrm{forma-}$ mide to HP25 $(<50 \mathrm{nM})$ that was substituted with different combinations of bases. After $10 \mathrm{~min}$ the reactions were placed on ice and analyzed by gel mobility shift assay. (T) Target only. (B) Hybridization of tiled 12-mer DNA probes to HP25. The protocol was identical to that described for the tiled LNA probes. 


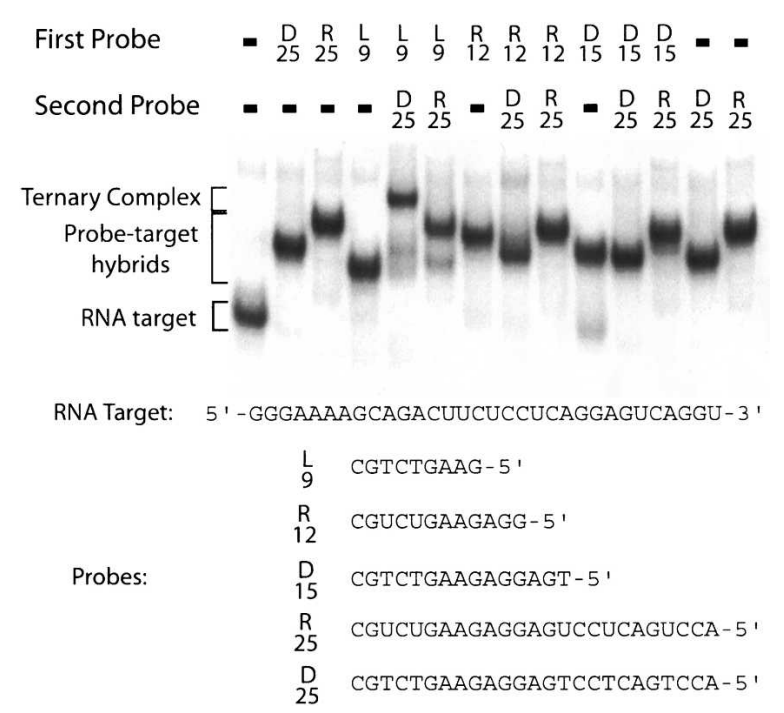

FIGURE 6. LNA in an RNA-LNA hybrid is displaced by RNA but not DNA. Hybridizations were conducted in $5 \mathrm{mM} \mathrm{MgCl}_{2}, 25 \mathrm{mM} \mathrm{NaCl}$, $20 \mathrm{mM}$ HEPES ( $\mathrm{pH} 7.5)$. The first probe $(5 \mu \mathrm{M})$ was annealed to the radiolabeled SS32 RNA target for $5 \mathrm{~min}$ at $60^{\circ} \mathrm{C}$. A second probe $(5$ $\mu \mathrm{M})$ was then added and annealing was continued for an additional $30 \mathrm{~min}$ at $37^{\circ} \mathrm{C}$. Reactions were kept on ice until analyzed by gel mobility shift assay.

lower temperatures, where the modified RNA might form secondary structures.

The combination of pseudo-complementary RNA targets and LNA probes could enable new technological advances in biomedical research. For example, due to the enhanced discrimination ability against mismatches, it should facilitate the detection of single nucleotide polymorphisms and point mutations. This combination could also promote the development of universal microarrays by improving the efficiency, specificity, and reliability of short probes. With every sequence in a target accessible, the sensitivity of such probes could increase by one or two orders of magnitude (Chou et al. 2004; Ramdas et al. 2004). The base analogs we have described should also help equalize the stability of hybrids that differ in G-C content (Gamper et al. 2004). In virtually all applications, specificity would require using $c G$ in place of hX. We have demonstrated that all three base analogs are incorporated into RNA by T7 RNA polymerase. However, while this enzymatic synthesis of pseudo-complementary RNA is convenient, it is currently limited to transcripts up to $200 \mathrm{nt}$. Further optimization of reaction conditions, together with engineering of T7 RNA polymerase for a broader range of nucleotide triphosphates, may remove this limitation. It is worth mentioning that we have used DNA polymerase to successfully incorporate nA, sT, and $\mathrm{hX} / \mathrm{cG}$ into long primer extension products $\left(>10^{3} \mathrm{nt}\right.$ in length; H.B. Gamper, A. Gewirtz, and Y.-M. Hou, unpubl. results), thus demonstrating the feasibility of using pseudocomplementary DNA as a target for short probes. Additional research is also needed to develop a pseudo-comple- mentary G-C base pair to allow hybridizations to be conducted under a broad range of conditions with short DNA probes.

\section{MATERIALS AND METHODS}

\section{Nucleic acids}

Oligonucleotides were purchased from Integrated DNA Technologies. Regular NTPs were from Roche Molecular Biochemicals, ITP was from Sigma, nATP, sUTP, and cGTP were from TriLink Biotechnologies, and $\left[\alpha-{ }^{32} \mathrm{P}\right] \mathrm{CTP}$ was from Amersham Biosciences. Transcription reactions contained 200-800 nM gel-purified dsDNA template with a consensus T7 promoter, $1 \mathrm{mM}$ ATP or nATP, 1 mM UTP or sUTP, $1 \mathrm{mM}$ GTP, ITP, or hXTP, $60 \mu \mathrm{M}\left[\alpha-{ }^{32} \mathrm{P}\right] \mathrm{CTP}$, $1 \mathrm{mM}$ GMP (only in the presence of a GTP analog), $6 \mathrm{mM} \mathrm{MgCl}$, $2 \mathrm{mM}$ spermidine, $10 \mathrm{mM}$ dithiothreitol, $0.5 \%$ Tween $20,40 \mathrm{mM}$ Tris- $\mathrm{HCl}$ at $\mathrm{pH} 7.9$, and T7 RNA polymerase (20 units; Ambion) in a total volume of $10-20 \mu \mathrm{L}$. After at least $5 \mathrm{~h}$ incubation at $37^{\circ} \mathrm{C}$, reactions were diluted to $50 \mu \mathrm{L}$ and centrifuged through a gel filtration column (CentriSpin 20, Princeton Separations). Radiolabeled transcripts were stored up to $6 \mathrm{wk}$ at $-20^{\circ} \mathrm{C}$. Chemically synthesized RNA oligomers were end labeled by T4 polynucleotide kinase reaction in the presence of $\left[\gamma-{ }^{32} \mathrm{P}\right] \mathrm{ATP}$, after which free counts were removed using a CentriSpin 10 column.

\section{Analysis of transcription reactions}

An aliquot of each transcription reaction was analyzed by electrophoresis in a $7 \mathrm{M}$ urea/12\% polyacrylamide gel. Full-length product was visualized and counted using a phosphorimager.

\section{Analysis of hybridization reactions}

The apparent melting temperature of each LNA-RNA hybrid was determined using a new gel mobility shift assay previously described (Gamper et al. 2004). Briefly, the hybrid of interest (containing a radiolabeled RNA) was subdivided into $10 \mu \mathrm{L}$ aliquots, each of which was incubated for $5 \mathrm{~min}$ at a different temperature within the range of $10^{\circ} \mathrm{C}-90^{\circ} \mathrm{C}$. The hybridization reactions were quenched by adding a 10 -fold molar amount of a competing oligonucleotide (which was identical in backbone composition and complementary to the probe) followed by rapid cooling in an ice bath. After the addition of a weighting solution, the reactions were loaded onto a nondenaturing $12 \%$ polyacrylamide gel prepared with $5 \mathrm{mM} \mathrm{MgCl} 2$ and electrophoresed in the cold room. After drying, gels were analyzed by phosphorimaging. Plotting the fraction of hybrid as a function of temperature yielded a melt curve and a $T_{m}$. A similar protocol was followed for determining the melting temperature of RNA hairpins. In this case the competing oligonucleotide (added in 100-fold molar excess) had an RNA backbone and was complementary to the entire length of one arm of the hairpin. Hybridization time courses were monitored by adding a competing oligonucleotide to reaction aliquots as a function of time. Tiling experiments, in which multiple probes were separately hybridized to RNA hairpin, were quenched in an ice bath with no prior addition of oligonucleotide competitor. 


\section{ACKNOWLEDGMENTS}

This study was supported by grants from the National Institutes of Health (CA-89325 to H.G., CA-72765 and CA-101859 to A.G., and GM-56662 to Y.-M.H.) and funds from the Doris Duke Charitable Foundation (to A.G.).

Received March 22, 2005; accepted June 9, 2005.

\section{REFERENCES}

Case-Green, S.C. and Southern, E.M. 1994. Studies on the base pairing properties of deoxyinosine by solid phase hybridisation to oligonucleotides. Nucleic Acids Res. 22: 131-136.

Chou, C.C., Chen, C.H., Lee, T.T., and Peck, K. 2004. Optimization of probe length and the number of probes per gene for optimal microarray analysis of gene expression. Nucleic Acids Res. 32: e99.

Doty, P., Boedtker, H., Fresco, J.R., Hall, B.D., and Haselkorn, R. 1959. Configurational studies of polynucleotides and ribonucleic acid. Ann. N. Y. Acad. Sci. 81: 693-708.

Gamper Jr., H.B., Gewirtz, A., Edwards, J., and Hou, Y.M. 2004. Modified bases in RNA reduce secondary structure and enhance hybridization. Biochemistry 43: 10224-10236.

Hacia, J.G., Woski, S.A., Fidanza, J., Edgemon, K., Hunt, N., McGall, G., Fodor, S.P., and Collins, F.S. 1998. Enhanced high density oligonucleotide array-based sequence analysis using modified nucleoside triphosphates. Nucleic Acids Res. 26: 4975-4982.

Howard, F.B. and Miles, H.T. 1984. 2NH2A $\times$ T helices in the riboand deoxypolynucleotide series. Structural and energetic consequences of 2NH2A substitution. Biochemistry 23: 6723-6732.

Jepsen, J.S., Sorensen, M.D., and Wengel, J. 2004. Locked nucleic acid: A potent nucleic acid analog in therapeutics and biotechnology. Oligonucleotides 14: 130-146.

Koshkin, A.A. 2004. Syntheses and base-pairing properties of locked nucleic acid nucleotides containing hypoxanthine, 2,6-diaminopurine, and 2-aminopurine nucleobases. J. Org. Chem. 69: 3711-3718.
Kumar, R.K. and Davis, D.R. 1997. Synthesis and studies on the effect of 2-thiouridine and 4-thiouridine on sugar conformation and RNA duplex stability. Nucleic Acids Res. 25: 1272-1280.

Lima, W.F., Monia, B.P., Ecker, D.J., and Freier, S.M. 1992. Implication of RNA structure on antisense oligonucleotide hybridization kinetics. Biochemistry 31: 12055-12061.

Martin, F.H., Castro, M.M., Aboul-ela, F., and Tinoco Jr., I. 1985. Base pairing involving deoxyinosine: Implications for probe design. Nucleic Acids Res. 13: 8927-8938.

Ramdas, L., Cogdell, D.E., Jia, J.Y., Taylor, E.E., Dunmire, V.R., Hu, L., Hamilton, S.R., and Zhang, W. 2004. Improving signal intensities for genes with low-expression on oligonucleotide microarrays. BMC Genomics 5: 35.

Rosenbohm, C., Pedersen, D.S., Frieden, M., Jensen, F.R., Arent, S., Larsen, S., and Kock, T. 2004. LNA guanine and 2,6-diaminopurine. Synthesis, characterization and hybridization properties of LNA 2,6-diaminopurine containing oligonucleotides. Bioorg. Med. Chem. 12: 2385-2396.

Seela, F. and Driller, H. 1985. Solid-phase synthesis of the self-com-

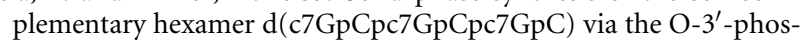
phoramidite of 7-deaza-2'-deoxyguanosine. Nucleic Acids Res. 13: 911-926.

Testa, S.M., Disney, M.D., Turner, D.H., and Kierzek, R. 1999. Thermodynamics of RNA-RNA duplexes with 2- or 4-thiouridines: Implications for antisense design and targeting a group I intron. Biochemistry 38: 16655-16662.

Vormbrock, R., Morawietz, R., and Gassen, H.G. 1974. Codon-anticodon interaction studies with trinucleoside diphosphates containing 2-thiouridine, 4-thiouridine, 2,4-diethiouridine, or 2-thiocytidine. Biochim. Biophys. Acta 340: 348-358.

Wallace, R.B., Shaffer, J., Murphy, R.F., Bonner, J., Hirose, T., and Itakura, K. 1979. Hybridization of synthetic oligodeoxyribonucleotides to phi chi 174 DNA: The effect of single base pair mismatch. Nucleic Acids Res. 6: 3543-3557.

Zoller, M.J. and Smith, M. 1983. Oligonucleotide-directed mutagenesis of DNA fragments cloned into M13 vectors. Methods Enzymol. 100: 468-500.

Zuker, M. 2003. Mfold web server for nucleic acid folding and hybridization prediction. Nucleic Acids Res. 31: 3406-3415. 

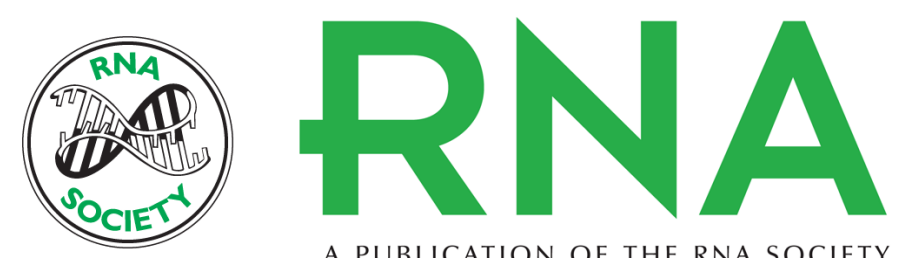

A PUBLICATION OF THE RNA SOCIETY

\title{
Unrestricted accessibility of short oligonucleotides to RNA
}

\author{
HOWARD B. GAMPER, JR., KHALIL ARAR, ALAN GEWIRTZ, et al.
}

RNA 2005 11: 1441-1447

\section{License}

Email Alerting Receive free email alerts when new articles cite this article - sign up in the box at the Service top right corner of the article or click here.

To subscribe to $R N A$ go to:

http://rnajournal.cshlp.org/subscriptions 